\title{
RNA-Based Mechanisms of Gene Silencing
}

\section{Contents}

6.1 The Unusual Behavior of Transgenes Led to the Discovery of Novel RNA-Based Silencing

Mechanisms - 118

6.1.1 Conserved Components of RNA-Based Silencing Mechanisms - 120

6.2 Post-Transcriptional Gene Silencing (PTGS) - 120

6.2.1 The Biogenesis and Function of microRNAs - 121

6.2.2 Genome Defense by siRNA-Mediated Silencing - 124

6.3 Transcriptional Gene Silencing (TGS) -125

6.4 Paramutation - 127

6.4.1 The cis-Regulatory Elements Controlling Paramutation and trans-Acting Factors Link Paramutation to RdDM - 129

References - 131 


\section{What You Will Learn in This Chapter}

Although epigenetic states are typically associated with DNA-methylation and posttranslational histone modifications, RNAs often play an important role in their regulation. Specific examples have already been discussed in the context of dosage compensation (see book Chap. 4 of Wutz) and genomic imprinting (see book Chap. 5 of Grossniklaus). In this Chapter, we will take a closer look at a particular class of RNAs implicated in gene silencing. Although the focus will lie on RNA-based silencing mechanisms in plants, many of its components, such as RNase III-related DICERLIKE endonucleases or small RNAbinding ARGONAUTE proteins, are conserved in animals, plants, and fungi. On the one hand, small RNAs are involved in post-transcriptional silencing by targeting mRNAs for degradation or inhibiting their translation, a feature that has been exploited for large-scale genetic screens. On the other hand, they also play a central role in transcriptional gene silencing, for instance in the repression of transposable elements across a wide variety of organisms. In plants, this involves a complex system whereby small RNAs derived from transposons and repeats direct DNA-methylation and repressive histone modifications in a sequence-specific manner. Recent results link this so-called RNA-dependent DNAmethylation to paramutation, a classical epigenetic phenomenon where one allele directs a heritable epigenetic change in another.

\subsection{The Unusual Behavior of Transgenes Led to the Discovery of Novel RNA-Based Silencing Mechanisms}

With the establishment of transformation technologies and their increasing use in plants in the late 1980s, it became apparent that the expression behavior of transgenes was hard to predict. While transgenics that did not show the expected expression of the transgene were initially discarded, they soon became an object of interest. One of the most striking examples was the characterization of transgenic petunia (Petunia hybrida) overexpressing the chalcone synthase (CHS) gene by Carolyn Napoli and Richard Jorgensen (Napoli et al. 1990). CHS encodes the rate-limiting enzyme of anthocyanin biosynthesis, which leads to the purple pigmentation of the flowers (• Fig. 6.1). Unexpectedly, instead of producing deep purple flowers due to CHS overexpression, many transgenics produced white flowers or flowers with a combination of purple and white tissues. Molecular analyses showed that the transgene had an effect on the endogenous CHS gene in trans, and that the transgene and the endogenous CHS gene were coordinately repressed (• Fig. 6.1). This phenomenon was termed "co-suppression". Subsequently, a similar observation was reported in the fungus Neurospora crassa, where the introduction of two transgenes of the carotenoid biosynthesis pathway led to a reduction in the expression of the endogenous gene, referred to as "quelling" (Romano and Macino 1992). RNA-based silencing was also discovered in animals, where the injection of RNA being either sense or antisense to the par-1 mRNA in the nematode Caenorhabditis elegans led to degradation of the par-1 transcripts (Guo and Kemphues 1995). Unlike the chromatin-based mechanisms described in earlier chapters, in these cases, gene silencing occurred at the post-transcriptional level (PTGS). Such cytoplasmic mechanisms can also contribute to the establishment of heritable epigenetic states as the RNAs involved are sometimes amplified in a self-sustaining fashion. Other RNA-based gene silencing 

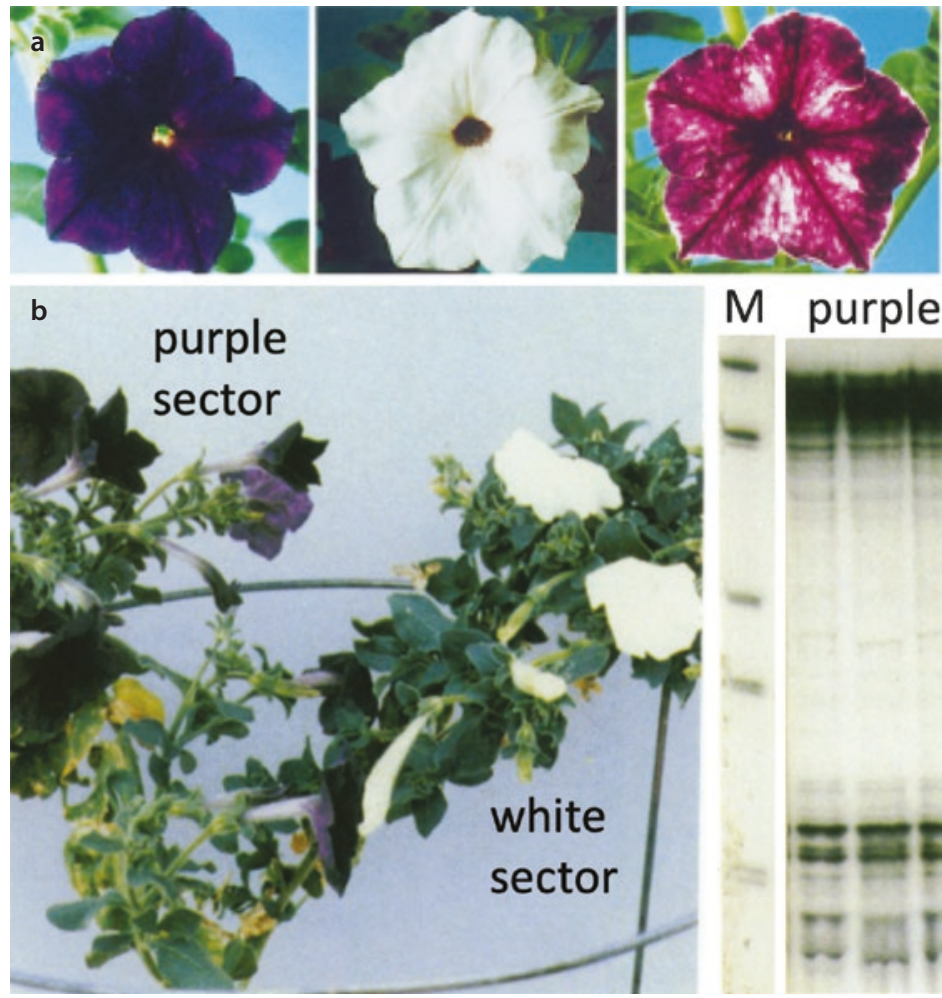

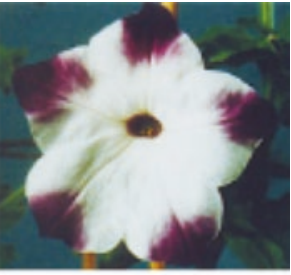

white

TG

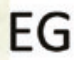

Fig. 6.1 Discovery of RNA-based gene silencing in petunia. a The wild type produces purple flowers due to anthocyanin production requiring the $C H S$ biosynthetic gene (left panel). Introduction of a CHS transgene leads to a variety of phenotypes, including completely white flowers (three panels on the right). (From Stam et al. 1997). b Some of these phenotypes are unstable, producing revertant sectors (left panel). RNAse protection assays showed that both transgene (TG) and endogenous CHS gene (EG) are expressed in the purple sectors but RNA levels of both TG and EG are strongly reduced in the white sectors (right panels). Three flowers per sector were analyzed; M, marker lane. (From Napoli et al. 1990)

phenomena in plants (Matzke et al. 1989) and animals (Bestor et al. 1994) occur in the nucleus and involve transcriptional gene silencing (TGS) by DNA methylation and chromatin modifications. It was soon clear that the various gene silencing phenomena were based on homology, i.e., showed sequence-specificity, and models involving DNA-DNA, DNA-RNA, and RNA-RNA pairing were proposed.

The seminal experiments of Fire and Mello (Fire et al. 1998) in C. elegans showed that not single-stranded but rather double-stranded RNA (dsRNA) was responsible for silencing, providing a unifying explanation for co-suppression, quelling, and other PTGS phenomena, now referred to as RNA interference (RNAi). In both plants and nematodes, gene silencing was heritable, suggesting a stable silencing intermediate. Hamilton and Baulcombe (1999) showed that this was not the fulllength dsRNA but rather short fragments of roughly $25 \mathrm{nt}$ in length, so-called short interfering RNAs (siRNAs). Similar 21-23 nt long siRNAs were then also found to be involved in RNAi in Drosophila melanogaster and C. elegans. The demonstration that chemically synthesized 21-22 nt dsRNAs can downregulate target mRNAs also 
in mammalian cells, opened completely new avenues for functional studies in cell culture. These discoveries did not only stimulate intensive research into the underlying mechanisms but also led to a wide range of applications (Sen and Blau 2006).

\subsubsection{Conserved Components of RNA-Based Silencing Mechanisms}

Given that siRNAs are involved in diverse silencing mechanisms in animals and plants, it is not surprising that they also use similar proteins that mediate the function of siRNAs. As the mechanisms are diverse, only the two key components are introduced here: the enzymes responsible for dicing the dsRNA into the siRNAs [DICERLIKE (DCL) proteins] and the ones involved in slicing the target mRNAs [ARGONAUTE (AGO) proteins]. DCL and AGO proteins are central to essentially all RNA-based silencing mechanisms.

DCL proteins, named after the founding member Dicer, were identified in $D$. melanogaster through a candidate gene approach testing different RNase III family members for the ability to cleave dsRNA into siRNAs (Bernstein et al. 2001). DCL proteins have a conserved structure, with two RNase III domains that cleave the dsRNA, a PAZ domain binding the 3'end of the dsRNA, and a helicase domain that unwinds the dsRNA duplexes (• Fig. 6.2). In some species, e.g., the model plant Arabidopsis thaliana, the family diversified and different members have specialized functions.

Another family of proteins involved in various silencing pathways are the ARGONAUTE (AGO) proteins, which are the catalytic subunit of an RNA-induced silencing complex (RISC) that slices the target mRNAs. Like DCL proteins, AGOs have a PAZ domain that binds the 3'end of dsRNAs, in this case the siRNAs produced by DCL proteins, which can base-pair with complementary target mRNAs. Through their PIWI domain, AGOs can slice the target mRNA complementary to the siRNA (- Fig. 6.3). However, cleaving the mRNA is not the only outcome of base-pairing with the siRNA; depending on the silencing pathway and type of RISC, translation of the mRNA can be blocked instead. AGO proteins can also be incorporated into another complex, called RNA-induced transcriptional silencing (RITS) complex, which is targeted to homologous DNA regions. The RITS complex then recruits enzymes that mediate DNA methylation and/or histone modifications which are associated with TGS.

\subsection{Post-Transcriptional Gene Silencing (PTGS)}

There is a wide variety of small RNAs that are involved in targeting mRNAs for degradation or inhibiting translation at the post-transcriptional level (PTGS). Others are involved in the sequence-specific silencing of genes at the transcriptional level (TGS) by establishing repressive chromatin (Pikaard and Mittelsten Scheid 2014). These small RNAs can be derived from transgenes or viruses (siRNAs) but many of them are endogenously produced during the life cycle of the plant, including microRNAs (miRNAs), hairpin siRNAs (hp-siRNA), trans-acting siRNAs (tasiRNAs), 
Arabidopsis DCL1

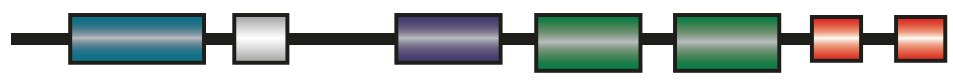

Arabidopsis DCL2

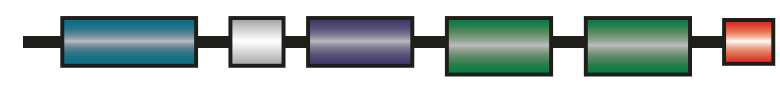

Arabidopsis DCL3

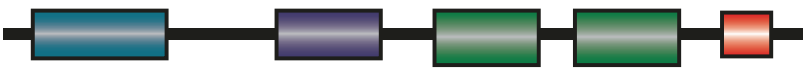

Arabidopsis DCL4

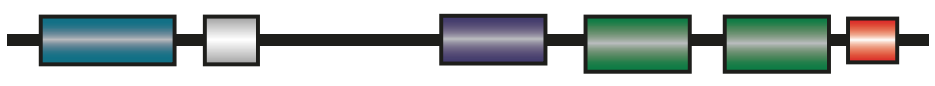

C. elegans Dicer

Drosophila Dicer-1
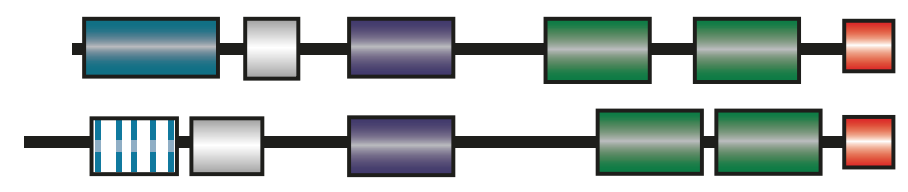

Drosophila Dicer-2

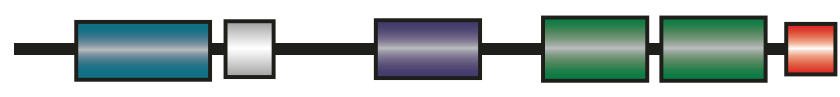

mouse Dicer

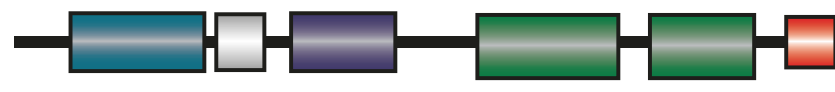

human Dicer

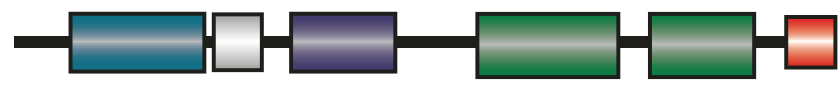

RNase III domain

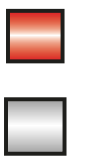

dsRNA binding domain

PAZ domain

helicase domain

\#I truncated helicase domain

Fig. 6.2 Domain organization of DICERLIKE (DCL) proteins from various eukaryotes. DCL proteins are responsible for dicing dsRNAs of diverse origin, e.g., derived from transgenes, endogenous genes, or viruses, into siRNAs. They are also crucial for the biosynthesis of microRNAs (miRNAs), regulatory small RNAs produced from stem-loop structures formed by transcripts of endogenous loci. The RNase III domains are responsible for dicing, while other domains are involved in binding the target dsRNA. (From Johanson et al. 2013)

phased siRNAs (phasi RNAs) produced during anther development, and epigenetically activated siRNA (easiRNA) that accumulate in the sperm cells (• Fig. 6.4). Here, we will look at the biogenesis and function of only a few selected types of small RNAs (Borges and Martienssen 2015).

\subsubsection{The Biogenesis and Function of microRNAs}

In both animals and plants, miRNAs play important roles in development by limiting the function of their target mRNAs to specific cells or tissues through their degradation or translational repression. Thus, they regulate gene expression at the post-transcriptional level and have an effect as long as they are present in the same 

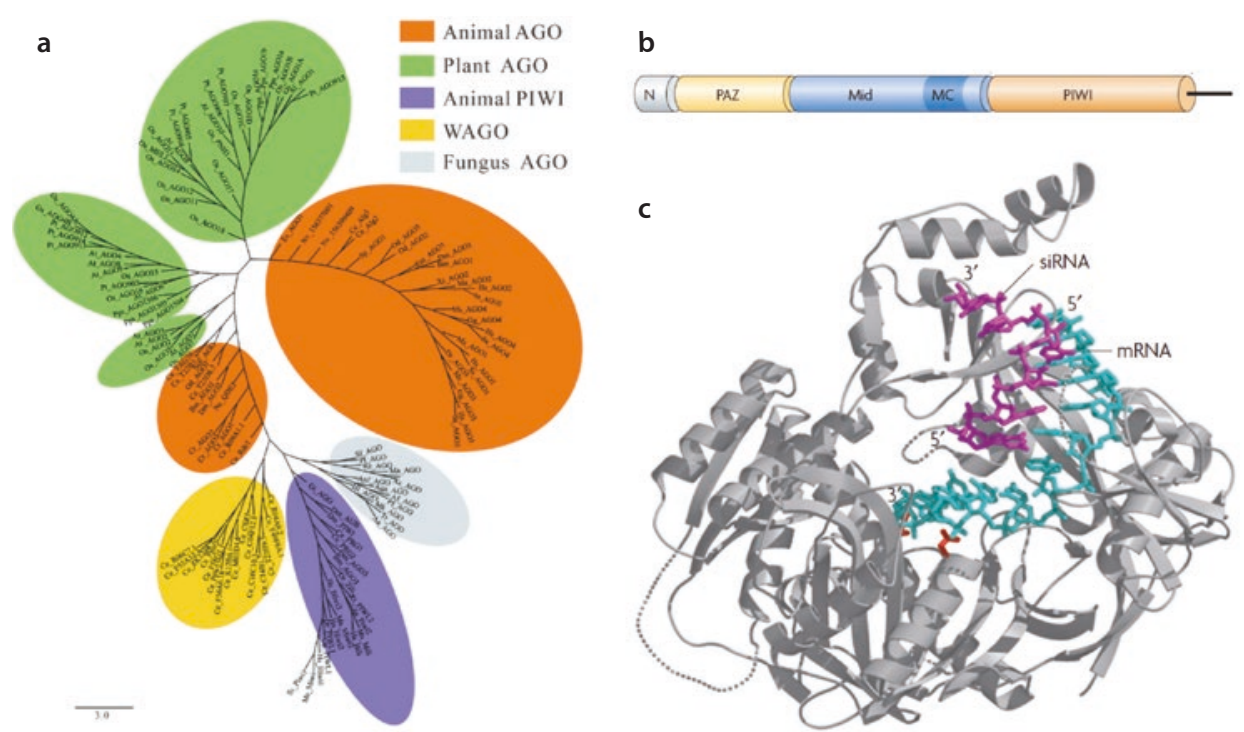

- Fig. 6.3 Phylogenetic tree and domain structure of ARGONAUTE (AGO) proteins. a Unrooted phylogenetic tree of 135 AGO proteins. The AGO proteins fall into three major clades: AGO proteins from plants, animals, and fungi that bind miRNAs or siRNAs, PIWI proteins which bind to Piwi-interacting RNAs (piRNAs) expressed in the germline of animals only, and the worm-specific WAGO clade. In some species, this family is highly diversified, e.g., in A. thaliana with 10 and C. elegans with 25 members, respectively. (From Wei et al. 2013). b Domain structure of AGO proteins with a PAZ domain that binds siRNAs, the PIWI domain that cleaves the target mRNA, and the cap-binding-like domain MC that lies within the Mid-domain connecting the PAZ and PIWI domains. (From Hutvagner and Simard 2008). c Crystal structure of an AGO protein, showing the 3 'end of the siRNA in purple and part of the mRNA in turquoise; active residues of the PIWI domain are shown in red. (From Song et al. 2004)

cell as the target mRNA. Because they do not result in a heritable change of gene expression, they are often not considered epigenetic regulators. An epigenetic change in gene expression persists even if the initial trigger, be it developmental or environmental, is gone (see also book $>$ Chap. 3 of Paro). Nevertheless, because their biogenesis and function are similar to epigenetically acting, short RNAs whose level is sustained, e.g. by RNA-dependent RNA polymerases (RDRs) that generate dsRNAs in a feed-forward loop, they are also discussed here.

miRNAs are derived from RNA polymerase II (Pol II) transcribed genes that do not encode proteins, but they can also originate from sequences in long non-coding RNAs or introns of protein-coding genes. These precursor RNAs have stretches of self-complementarity and can form imperfect RNA hairpins or stem-loop structures, which are then processed into the miRNAs. In mammals, these pri-miRNAs are cleaved by the RNase III-type endonuclease Drosha on one side of the loop in the nucleus. The resulting partially processed pre-miRNA is then transported to the cytoplasm where Dicer cuts the stem $22 \mathrm{nt}$ away from the Drosha cleavage site to generate an RNA duplex consisting of the miRNA and its complementary strand. In plants, both cleavages are made by DCL1 in the nucleus and result in $21 \mathrm{nt}$ long miRNA duplexes ( $\bullet$ Fig. 6.4). Before these are exported to the cytoplasm, they are processed by HUA ENHANCER1 (HEN1), which methylates the 3'-terminal ribose of each RNA strand to protect the miRNA from degradation. The miRNA is then 
a

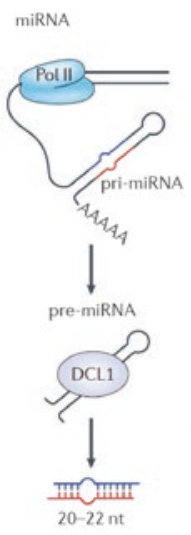

PTGS

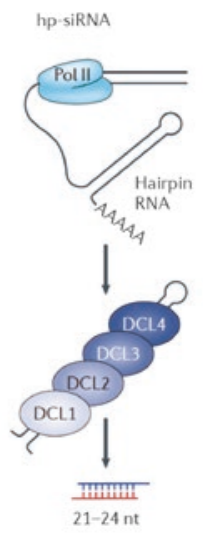

natsiRNA
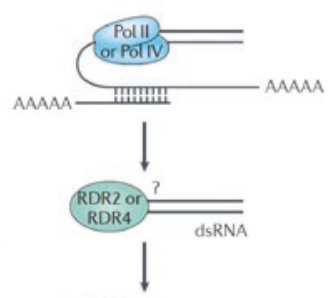

DCL1, DCL2

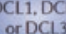

or DCl 3
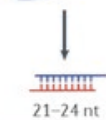

cis-natsiRNA or trans-natsiRNA

mRNA cleavage and translational repression

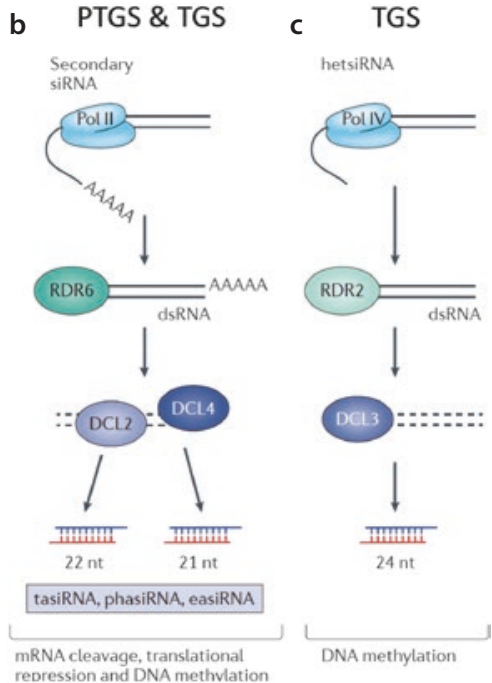

b

epression and DNA methvlation

- Fig. 6.4 Different small RNA biogenesis pathways in plants. a Pathways involved in PTGS: transcripts from miRNA genes form hairpin-like structures (pri-miRNA) that are diced by DLC1 into shorter stem-loop structures (pre-miRNA) and then into mature miRNA duplexes. Longer hairpin structures can originate from inverted repeats and are processed by all DCL proteins into hp-siRNAs. They may represent an early stage in the evolution of miRNAs. natsiRNAs are derived from dsRNA produced by overlapping transcription (cis-natsiRNAs) or from transcripts with regions of high complementary originating from distant loci (trans-natsiRNAs). RNA-dependent RNA polymerases (RDRs) are likely involved in extending the dsRNA, which is diced by various DCLs. b Secondary siRNAs act on transcripts distinct from those that generated them and are involved in both PTGS and TGS. The transcripts come from non-coding loci, protein-coding genes, or transposons and are converted into dsRNA by RDR6 and processed by DCL2 and DCL4 into various classes of siRNAs. The tasiRNAs, for instance, are derived from non-coding transcripts of specific TAS genes (TAS1 to TAS4 in $A$. thaliana), which are targeted by specific miRNAs that mediate their cleavage (e.g., miR173-AGO1 for TAS1/TAS2; mir390-AGO7 for TAS3). The 3' cleavage product is converted into dsRNA by RDR6, and then sequentially diced by DCL4 into $21 \mathrm{nt}$ long tasiRNAs that are phased relative to the miRNA cleavage site. Thus, tasiRNAs have defined sequences and function like miRNAs to slice or inhibit their target mRNAs. c The heterochromatic siRNAs (hetsiRNAs) originate from transposons and repeats, often located in pericentromeric regions. They depend on transcription by RNA polymerase IV (Pol IV), dsRNA synthesis by RDR2, and dicing into 24 nt long siRNAs by DCL3. hetsiRNAs mediate RNA-dependent DNA methylation (RdDM). (From Borges and Martienssen 2015)

bound by $A$. thaliana AGO1 (sometimes AGO7/AGO10) to form a RISC complex that slices the target mRNA or blocks its translation. In plants, the miRNA typically shows near-perfect complementarity to the target mRNA, leading to slicing in the majority of cases. In contrast, animal miRNAs rarely have perfect complementarity to the mRNA and more often cause translational inhibition. Also in plants, some miRNA block translation in combination with slicing of the transcript. Many plant miRNAs target transcription factors and are often conserved during evolution, indicating important regulatory roles during development. Although the coding sequences for these transcription factors have diverged considerably, the target sites on the mRNAs matching the miRNAs are nearly invariant. Other miRNAs are evolutionary young and often specific to a cell lineage. In A. thaliana, these lineage-specific miRNAs are sometimes processed by DCL4, while DCL1 is required for the vast majority of miRNAs. 


\subsubsection{Genome Defense by siRNA-Mediated Silencing}

An important class of small RNAs are the siRNAs that induce PTGS to defend the genome against viruses and microbes (Pikaard and Mittelsten Scheid 2014). Most plant viruses have a ssRNA genome whose replication depends on an endogenous or virus-encoded RDR to produce dsRNA intermediates, which are substrates for DCL proteins. In addition, viruses with dsRNA genomes are direct substrates for dicing as are dsRNA intermediates formed during the life cycle of ssDNA viruses. The silencing of transgenes is likely observed due to the activity of similar genome defense pathways. Transgenes often integrate as multiple copies that can have opposite orientations. Transcription through inverted transgenes can lead to hairpin-like RNA structures that are substrates for DCL proteins. Transgenes may also integrate next to strong promoters pointing in the opposite direction, leading to transcription of both strands and, thus, dsRNA formation. Finally, other aberrant types of transgene-derived mRNAs can serve as a substrate for an RDR protein, generating dsRNA. PTGS of viruses and transgenes typically involves 21-22 nt long siRNAs generated primarily by DCL2 and DCL4 (• Fig. 6.4). The $21 \mathrm{nt}$ long siRNAs guide the cleavage of the mRNA by AGO1, which generates a $3^{\prime}$ fragment of the mRNA that lacks a 7-methylguanosine cap at its 5'end. mRNAs without a cap are eliminated rapidly by exonuclease-mediated degradation. The association of $22 \mathrm{nt}$ siRNAs with AGO1 couples target mRNA cleavage to the recruitment of RDR6, such that the 3' cleavage fragment is transcribed into dsRNA, which is then diced again by DCL4 to make $21 \mathrm{nt}$ long secondary siRNAs. DCL3 can also dice dsRNA derived from viruses or transgenes, generating $24 \mathrm{nt}$ siRNAs (• Fig. 6.4). However, these lead to TGS through DNA and chromatin modifications at loci with complementarity to the siRNAs. Taken together, these diverse aspects induced by siRNAs mount a very powerful response to invading nucleic acids. Not surprisingly, viruses developed a variety of countermeasure and many encode suppressors of gene silencing in their genomes (Jiang et al. 2012).

The discovery of RNAi and its underlying mechanisms, many of which are conserved across organisms, have opened unprecedented opportunities for applications. For instance, the discovery that synthetic dsDNA can be used in tissue culture cells to induce RNAi allowed genome-wide screens to address gene function in cultured cells. This has been instrumental not only for basic research but especially for drug discovery in the pharmaceutical industry (Hokaiwado et al. 2008). In plants, miRNAs have been used to address the function of genes or gene families by introducing artificial miRNAs that target transcripts derived from one or multiple loci. Also, siRNAs derived from transgenic hairpin constructs that target a specific gene of interest have been extensively used to study gene function. Moreover, viral genomes have been engineered to encode plant genes of interest. A viral infection thereby leads to the production of siRNAs against the endogenous plant gene, allowing functional studies. This so-called virus-induced gene silencing (VIGS) is particularly useful to study the function of genes in non-model organisms, where no efficient transgenesis methods are available but infections using such engineered viruses are possible.

Similar approaches have led to biotechnological applications. One of the most successful advancements was the development of virus-resistant plants by exploiting 

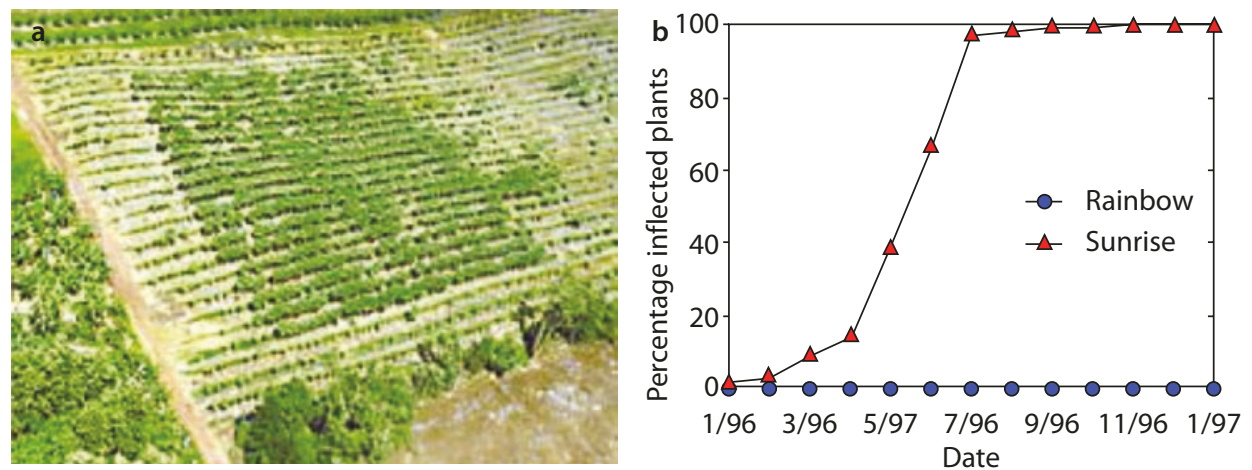

Fig. 6.5 Virus-resistant, transgenic papaya based on RNAi directed against the coat protein of papaya ringspot virus. a Field trial showing a block of resistant papaya plants of the transgenic Rainbow cultivar surrounded by susceptible non-transgenic plants of the Sunrise cultivar. b All transgenic Rainbow plants were resistant to the virus while all non-transgenic Sunrise plants became infected within a few months of the field trial. (From Gonsalves et al. 2004)

the siRNA-based defense mechanism of the plant and targeting it specifically to the virus. For instance, the Hawaiian production of papaya was severely threatened by papaya ringspot virus, which devastated the plantations in the 1990s. The introduction of a genetically modified virus-resistant papaya variety in 1998 saved the papaya growers and is the basis of current papaya production in the USA (• Fig. 6.5). A similar approach has since been used to protect commercially grown summer squash (Gottula and Fuchs 2009).

\subsection{Transcriptional Gene Silencing (TGS)}

Transgene silencing does not only involve siRNAs at the post-transcriptional level but also comprises DNA methylation. The two phenomena were linked when it was discovered that homology-dependent DNA methylation also involved siRNAs (Mette et al. 2000) and some mutants of $A$. thaliana affected both PTGS and TGS. Furthermore, the silencing of transposable elements and repeats is crucial for the maintenance of genome integrity. A failure to keep transposons in check results in hybrid dysgenesis in $D$. melanogaster, where massive transposition in the germline leads to sterility, a high mutation rate, and chromosome breakage. In metazoans, transposons are silenced through the action of Piwi-interacting RNAs (piRNAs), which are generated from long Pol II transcripts that are derived from piRNA clusters, loci composed of transposons of diverse families or fragments thereof. Thus, piRNAs are complementary to many dispersed transposons. Their production is independent of DCL proteins but piRNAs bind to AGO-like proteins of the PIWI clade ( $\bullet$ Fig. 6.3), which are essential to their biogenesis. Primary piRNAs can be amplified through a so-called ping-pong cycle and eventually lead to silencing of the transposons by recruiting the machinery to establish H3K9me3 and, depending on the organism, de novo DNA methyltransferases (Ernst et al. 2017). 
The silencing of endogenous repeats, such as those present at centromeres, was also found to rely on RNA-based mechanisms, for instance in the formation of centromeric heterochromatin in A. thaliana or the yeast Schizosaccharomyces pombe (Martienssen and Moazed 2015). Here, however, we focus on RNA-dependent DNAmethylation ( $\mathrm{RdDM}$ ), an RNA-based mechanism primarily targeting endogenous transposons and repeats, but also transgenes, in plants (Matzke and Mosher 2014).

RdDM was initially discovered in plants that were infected with a viroid (Wassenegger et al. 1994). It was later shown to also occur at transcriptionally silenced transgenes and rely on siRNAs and proteins of the RNAi machinery. RdDM is a very complex mechanism, involving many factors that were identified in genetic screens for mutants that relieve transgene silencing. In addition, the biochemical isolation of proteins interacting with known factors by co-immunoprecipitation contributed to complete the picture. Taken together, these studies revealed that RdDM relies on two specialized RNA polymerase complexes that are derived from Pol II, called Pol IV and Pol V. Conceptually, the RdDM mechanism can be separated into three major phases (• Fig. 6.6) (Matzke and Mosher 2014).

First, Pol IV is recruited to target loci by SAWADEE HOMEODOMAIN HOMOLOGUE1 (SHH1), which binds to H3K9me. The transcripts produced by Pol IV are then copied by RDR2, which interacts with the chromatin remodeler CLASSY1, into dsRNA that is diced into $24 \mathrm{nt}$ siRNAs by DCL3. The siRNAs are protected from degradation through methylation by HEN1 and incorporated into AGO4.

In a second phase, Pol V mediates de novo DNA methylation of the target loci by transcribing a scaffold RNA with complementarity to the AGO4-bound siRNAs. ChIP-sequencing experiments showed that Pol V is targeted to transposons and

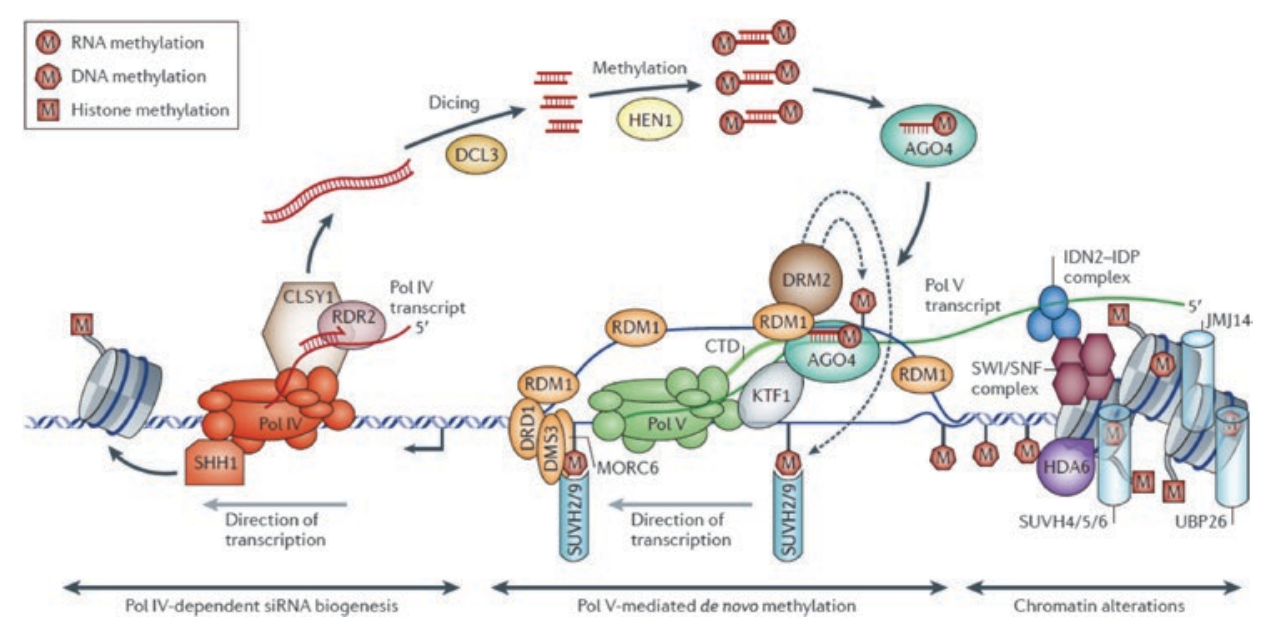

- Fig. 6.6 Schematic representation of the canonical RdDM pathway involving the plant-specific RNA polymerases Pol IV and Pol V. The three phases of establishing silent chromatin at target loci, mostly transposable elements and repeats but also transgenes, are shown. In phase one, 24 nt siRNA are generated through a Pol IV-dependent process (left). In the second phase, the target loci get de novo methylated through a Pol V-mediated mechanism (middle) and in the last phase, heterochromatin is established through repressive histone modifications, nucleosome repositioning, and changes in higher order chromatin structure (right). For details, see main text. (From Matzke and Mosher 2014) 
genomic repeats that are associated with DNA methylation and $24 \mathrm{nt}$ siRNAs. However, about $25 \%$ of the Pol V loci do not show these features, indicating that Pol $\mathrm{V}$ occupancy is not sufficient for RdDM. Pol V recruitment to target loci may involve interactions with SU(VAR)3-9 HOMOLOG2 (SUVH2) and SUVH9, which both bind to methylated DNA. Pol V transcription is likely facilitated through the unwinding of DNA by the chromatin remodeler DEFECTIVE IN RNA-DIRECTED DNA METHYLATION1 (DRD1) and the single strand DNA-binding ability of RNADIRECTED DNA METHYLATION1 (RDM1) that, together with DEFECTIVE IN MERISTEM SILENCING3 (DMS3) and MICROCHIDIA6 (MORC6), may establish and maintain the unwound state. AGO4 is recruited to the locus through interactions with KOW DOMAIN-CONTAINING TRANSCRIPTION FACTOR1 (KTF1) and binding to Pol V itself. The RDM1 protein brings the DOMAINS REARRANGED METHYLTRANSFERASE2 (DRM2) together with AGO4, leading to de novo DNA methylation of regions with homology to the siRNAs.

Finally, in the third phase, a repressive chromatin state is established. This involves the repositioning of nucleosomes through a SWI/SNF chromatin remodeling complex (see book $>$ Chap. 2 of Paro) that interacts with a complex composed the Pol V scaffold RNA-binding proteins INVOLVED IN DE NOVO2 (IND2) and IND2 PARALOGUE (IDP). Active histone marks are removed by HISTONE DEACETYLASE6 (HDA6), histone demethylase JUMONJI14 (JMJ14), and UBIQUITIN-SPECIFIC PROTEASE26 (UNP26), followed by the deposition of repressive histone modifications, e.g., H3K9me, by SUVH4, SUVH5, and SUVH6. The silent state may be further reinforced through changes in higher order chromatin conformation mediated by the activity of MORC1 and MORC6.

Mutations affecting the RdDM machinery are viable and fertile and do typically not show gross developmental aberrations. However, the pathway is important for transposon repression and, thus, contributes to maintaining the integrity of the genome. Furthermore, it plays a role in various physiological and developmental processes (Matzke and Mosher 2014). Thus, RdDM has been implicated in the defense against bacteria and viruses. Additionally, it may be involved in establishing some gametic imprints (see book $>$ Chap. 5 of Grossniklaus). Moreover, siRNAs can be transported over short distances from cell to cell but also long-distance through the vasculature, potentially playing a role in intercellular communication. This has for instance been proposed to occur between cells in the female gametophyte and pollen, and may also occur between embryo and endosperm, although direct evidence for the transport of siRNAs in these tissues is missing. Finally, RdDM has been shown to occur in hybrids, where the production of siRNAs from one parental genome can affect previously non-methylated loci in the other genome in trans, either at allelic sites or at distant loci if there is sufficient sequence homology.

\subsection{Paramutation}

Paramutation is a classical epigenetic phenomenon that is now understood to involve RdDM. It was simultaneously discovered by Alexander Brink and Edward Coe in maize as well as Robert Hagemann in tomato (Brink 1956; Coe Jr 1959; Hagemann 1958). Paramutation describes a paradox of genetics where certain alleles do not show the expected segregation pattern based on the rules of Mendel. The discoverers 
found that a 'genetic' change or 'conversion' occurred in certain heterozygous combinations. For instance, Coe worked with alleles of the boosterl (bl) locus responsible for anthocyanin pigmentation of the plant and reported "an effect on plant color in maize, interpreted as a regular conversion of one allele at the $B$ locus by another [...]. The effect is a continuing one in that the 'converted' $B$, termed $B$ ', is also regularly able to 'convert' newly introduced $B^{\prime}$. When Coe crossed intensely pigmented, purple plants homozygous for the boosterl-Intense $(B-I)$ allele to weakly pigmented, green plants homozygous for the $B$ allele, all progeny was weakly pigmented. This could, in principle, be explained by dominance of $B$ over $B-I$, but the progeny also remained weakly pigmented after repeated outcrossing to $B$ - $I$ plants. Thus, given there was no segregation, the $B-I$ allele appeared to have changed its activity to that of the weakly pigmented $B$ allele and was termed $B^{\prime}(\bullet$ Fig. 6.7). The non-Mendelian segregation was confirmed in test-crosses of $B-I / B$ heterozygotes to unpigmented plants homozygous for the loss-of-function $b$ allele. Instead of the expected 1:1 segregation of intensely pigmented $B-I / b$ and weakly pigmented $B / b$ plants, all progeny was weakly pigmented, confirming the conversion of $B-I$ to $B^{\prime}$. Already in these early studies, Coe found that the $B-I$ allele can spontaneously give rise to the $B^{\prime}$ allele and Brink found the altered state of the $R^{\prime}$ allele of the coloured $1(r I)$ locus to revert in some of the progeny. Such instability or metastability is now considered a hallmark of an epigenetic state, which is much less stable than genetic mutations.

Nowadays, the conversion of one allele into another is understood as a change in the epigenetic control of the expression state of the locus and is called paramutation. Paramutation is defined as a directed, heritable change in gene expression that is invoked by interactions between specific alleles. Paramutation can occur between genetically identical or distinct alleles. The alleles that incite paramutation are called paramutagenic, those that are susceptible to it are paramutable, and those that do
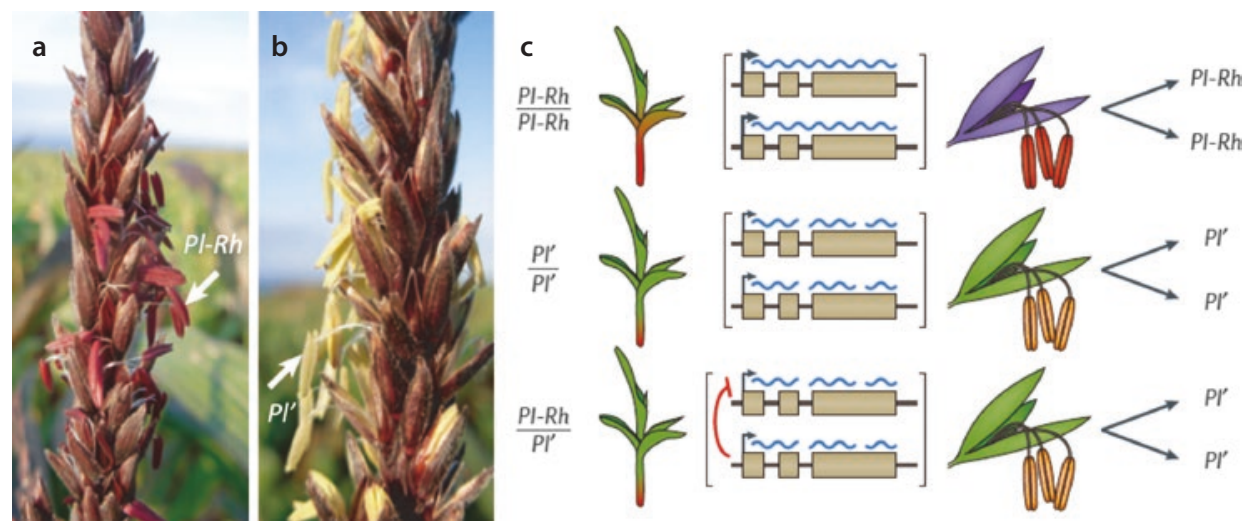

Fig. 6.7 Paramutation illustrated by the genetic behavior of the purple plant 1 -Rhoades $(P l-R h)$ allele required for anthocyanin pigmentation of the plant. a Pigmented anthers of a $P l-R h$ plant. b Unpigmented anthers of a Pl' plant. (a, b from Hollick 2017). c Schematic representation of paramutation at the $p l 1$ locus. Diploid genotypes and seedling coloration are shown on the left, anther color and haploid genotypes of the gametes produced are shown on the right. In the middle, transcription from the $p l l$ locus is represented: the strongly expressed $P l-R h$ allele, the weekly expressed $P l$ ' allele with broken wavy lines representing unstable mRNAs, and the interaction between the paramutagenic $P l^{\prime}$ and the paramutable $P l-R h$ (red arrow), leading to reduced expression of $P l-R h$ and conversion to $P l$ '. (From Hollick 2010) 
not participate in paramutation are neutral. As Coe described, the converted paramutable allele then becomes paramutagenic itself and can incite secondary paramutation. This interesting epigenetic phenomenon has been described for several endogenous loci in tomato and maize, including $b 1, r 1$, purple plant 1 (pll), and pericarp color1 ( $p 1)$, all which are transcriptional regulators of flavonoid biosynthesis. But there is also an increasing number of transgenes that show a paramutation-like behavior, both in plants and animals, including D. melanogaster, C. elegans, and mice (Hollick 2017).

\subsubsection{The cis-Regulatory Elements Controlling Paramutation and trans-Acting Factors Link Paramutation to RdDM}

Most paramutation systems in maize are metastable and the expression state of an allele can spontaneously change in both directions at variable frequencies. At the $b l$ locus, however, $B^{\prime}$ alleles are formed spontaneously from $B-I$, while the $B^{\prime}$ state is extremely stable. In genetic screens metastability can be problematic as it may generate false positives. The stability of the $B^{\prime}$ state was one of the reasons to use this system in various genetic screens to identify cis-regulatory regions and trans-acting factors of paramutation.

For instance, the cis-regulatory regions required for high expression of $B-I$ were localized by looking for recombinants where high expression was acquired by a neutral allele. Likewise, the cis-elements required for paramutation were identified by isolating recombinants where a neutral allele had become paramutagenic. Mapping of the recombination breakpoints showed that the enhancer for high $b 1$ expression and the region mediating paramutation coincided and were located about $100 \mathrm{~kb}$ upstream of the $b 1$ transcriptional start site (Stam et al. 2002). Molecular characterization of this region showed that it was composed of seven tandem repeats of an $853 \mathrm{bp}$ sequence that is unique in the maize genome (- Fig. 6.8). These repeats were identical in the paramutagenic $B^{\prime}$ and the paramutable $B$ - $I$ alleles, confirming the epigenetic nature of paramutation.

Paramutagenicity is related to how many copies of the repeat are present in an allele. With five repeats, an allele is still fully paramutagenic but with three, paramutagenicity is reduced, and neutral alleles have only a single repeat (• Fig. 6.8). A relationship between the number of repeats and paramutagenicity was also observed at the $r 1$ locus, where the paramutagenic alleles $R$-stippled and $R$-marbled both consist of multiple copies of the $r l$ coding region and flanking sequences, while alleles with only one $r l$ gene are neutral. Deletion derivatives showed a direct correlation between paramutagenicity and the number of $r l$ genes (Hövel et al. 2015).

Given that both $B-I$ and $B^{\prime}$ have the same seven repeats, the difference between them must lie in their epigenetic state. Indeed, at several loci, the paramutable and paramutagenic alleles were shown to be differentially methylated (• Fig. 6.8). Importantly, upon paramutation, the paramutable allele gained DNA methylation, implying methylation is associated with and possibly even triggering the epigenetic switch underlying paramutation. Thus, during paramutation, the cis-regulatory regions mediate trans-homolog interactions between the two alleles, leading to a 'transfer' of DNA methylation. The tandem repeats of $B$ ' are not only hypermethyl- 
a

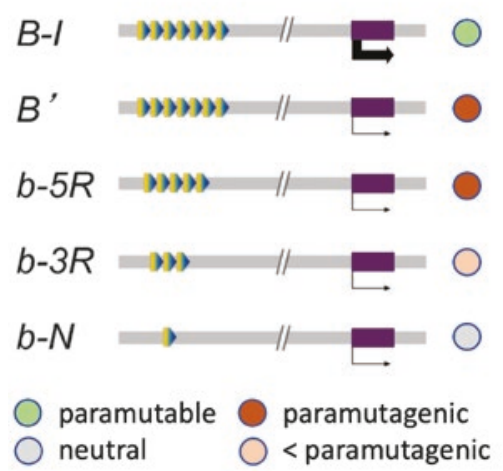

c
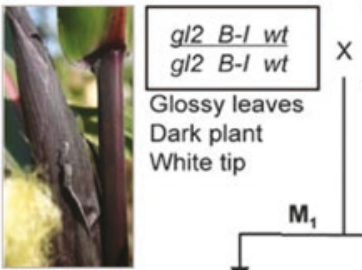

Glossy leaves

Dark plant

White tip

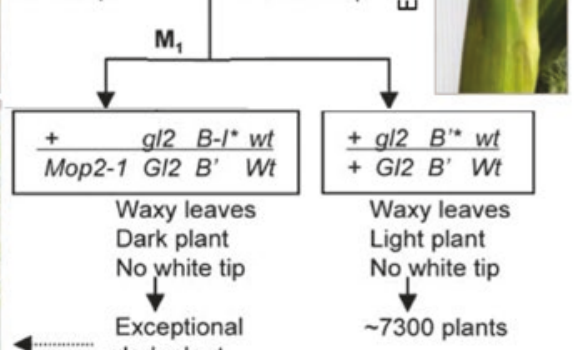

b
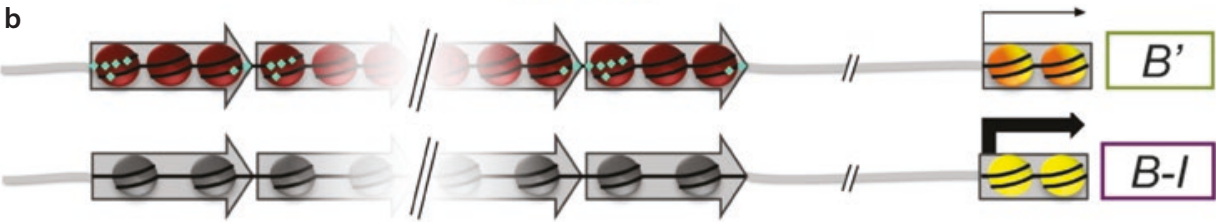

$1 /$
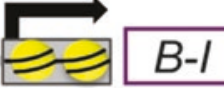

5-methyl
cytosine

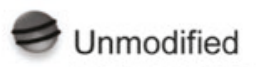
nucleosome

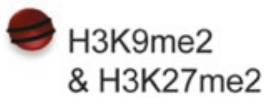

- Fig. 6.8 Features of cis-elements required for paramutation at the $b 1$ locus and genetic screen for trans-acting factors. a Repeat region $\sim 100 \mathrm{~kb}$ upstream of the $b 1$ coding sequence. The paramutagenic $B$ - $I$ and paramutable $B^{\prime}$ alleles have seven identical tandem repeats while the neutral $b-N$ allele has only one. A reduction to five repeats maintains full paramutagenicity while, with three repeats, the allele is less paramutagenic. b Epigenetic features of $B^{\prime}$ and $B$ - $I$, which show differential DNA methylation and carry distinct histone marks at the tandem repeats (only four repeats are show for simplicity) (a, b from Hövel et al. 2015). c Scheme of the genetic screen for Mediator of paramutation (Mop) mutants using EMS pollen mutagenesis. The two parental lines carry wild-type or mutant alleles of glossy2 (gl2) and white tip (wt), respectively, that flank the $b 1$ locus. $B^{\prime *}$ indicates a $B-I$ allele that was paramutated to $B^{\prime}$ in wild-type plants, $B-I^{*}$ indicates a $B-I$ allele exposed to $B^{\prime}$ in the presence of $M o p 2-1$, which prevents paramutation. (From Sidorenko et al. 2009)

ated as compared to those of $B-I$ but they also carry the repressive histone marks H3K9me2 and H3K27me3, similar to silenced transposons (• Fig. 6.8).

Using the powerful genetic tools in maize, screens for second-site mutations in factors required for the establishment and/or maintenance of paramutation were conducted (Hollick 2017). Looking for mutants that abolish the reduced pigmentation levels observed in $P l^{\prime} / P l^{\prime}$ and $B^{\prime} / B^{\prime}$ plants, parallel screens for required to maintain repression ( $\mathrm{rmr}$ ) and mediator of paramutation ( $\mathrm{mop}$ ) mutants were performed (- Fig. 6.8). The molecular cloning of some of these mutants provided a clear link to gene silencing by RdDM. The mopl locus was found to encode a homolog of RDR2, which is required for the generation of Pol IV-dependent 24 nt siRNAs in $A$. thaliana. Moreover, the rmr6/mop3 locus encodes the largest subunit of Pol IV, and rmr7/mop2 the second largest subunit, which is part of both Pol IV and Pol V, fully establishing the link between paramutation and RdDM. Indeed, all known mop and $r d r$ mutants do not only suppress the reduced pigmentation in $P l^{\prime} / P l^{\prime}$ plants but also fail to accumulate the corresponding $24 \mathrm{nt}$ siRNAs (Hollick 2017). However, the 
situation in maize is more complex than in A. thaliana as there are two non-redundant Pol IV and three Pol V subtypes. Thus, the specific roles of the RdDM components identified in maize through these screens are still not well understood. Clearly, there are some unique features of paramutation in comparison to transposon silencing by RdDM, namely the directed trans-homolog interactions and heritable changes occurring in meiosis that are a hallmark of paramutation. Future work will shed more light onto the similarities and differences of paramutation and RdDM.

\section{Take-Home Message}

- In diverse epigenetic phenomena, RNAs play a key role. These include long noncoding RNAs in genomic imprinting and $\mathrm{X}$ chromosome inactivation, or short RNAs of different types in gene silencing mechanisms.

- Gene silencing can occur at the transcriptional or post-transcriptional level but both mechanisms rely on short RNAs. The mechanisms play important roles in the defense against viruses as well as in maintaining genome integrity.

- Many of the components of RNA-based silencing mechanisms are conserved in animals, plants, and fungi. These include some key players like the RNase IIIrelated DICERLIKE endonucleases and the ARGONAUTE proteins that bind to small RNAs and slice target mRNAs.

- Small RNAs are involved in post-transcriptional silencing, targeting mRNAs for degradation or inhibiting their translation. This mechanism is also referred to as RNA interference (RNAi) and has been instrumental for large-scale screens in cultured cells both in fundamental and applied research.

- Transcriptional gene silencing, for instance to repress transposable elements and repeats, is also based on small RNAs - siRNAs or piRNAs - across a wide variety of organisms. In plants, this is achieved by RNA-dependent DNA-methylation, a complex pathway involving the plant-specific RNA polymerases Pol IV and Pol V.

- Paramutation involves trans-homolog interactions that cause a heritable repression of the paramutable allele directed by the paramutagenic allele. Similar effects were observed in hybrids where bringing together divergent (epi)genomes can cause DNA methylation changes in trans.

- Paramutation relies on cis-regulatory sequences that can be far away from the coding sequence and consist of repeats. The epigenetic state of these repeats differs between paramutable and paramutagenic alleles and is established and/or maintained by the RNA-dependent DNA methylation pathway.

\section{References}

Bernstein E, Caudy AA, Hammond SM, Hannon GJ (2001) Role for a bidentate ribonuclease in the initiation step of RNA interference. Nature 409:363-366. https://doi.org/10.1038/35053110.

Bestor TH, Chandler VL, Feinberg AP (1994) Epigenetic effects in eukaryotic gene expression. Genesis 15:458-462. https://doi.org/10.1002/dvg.1020150603

Borges F, Martienssen R (2015) The expanding world of small RNAs in plants. Nat Rev Mol Cell Biol 16:727-741. https://doi.org/10.1038/nrm4085

Brink RA (1956) A genetic change associated with the $R$ locus in maize which is directed and potentially reversible. Genetics 41:872-889. PMID: 17247669 
Coe EH Jr (1959) A regular and continuing conversion-type phenomenon at the $B$ locus in maize. Proc Natl Acad Sci USA 45:828-832. https://doi.org/10.1073/pnas.45.6.828

Ernst C, Odom DT, Kutter C (2017) The emergence of piRNAs against transposon invasion to preserve mammalian genome integrity. Nat Commun 8:1411. https://doi.org/10.1038/s41467-017-01049-7

Fire A, Xu S, Montgomery MK, Kostas SA, Driver SE, Mello CC (1998) Potent and specific genetic interference by double-stranded RNA in Caenorhabditis elegans. Nature 391:806-811. https://doi. org $/ 10.1038 / 35888$

Gonsalves D, Gonsalves C, Ferreira S, Pitz K, Fitch M, Manshardt R, Slightom J (2004) Transgenic virus resistant papaya: from hope to reality for controlling papaya ringspot virus in Hawaii. APSnet Feature Story July 2004. https://pdfs.semanticscholar.org/6db3/b62d6f8348fc780e4b5a3a8f293da309e52c.pdf?_ga $=2.17733369 .1394956602 .1601814746-498759226.1601380287$

Gottula J, Fuchs M (2009) Toward a quarter century of pathogen-derived resistance and practical approaches to plant virus disease control. Adv Virus Res 75:161-183. https://doi.org/10.1016/ S0065-3527(09)07505-8.

Guo S, Kemphues KJ (1995) par-1, a gene required for establishing polarity in C. elegans embryos, encodes a putative Ser/Thr kinase that is asymmetrically distributed. Cell 81:611-620. https://doi. org/10.1016/0092-8674(95)90082-9

Hagemann R (1958) Somatische Konversion bei Lycopersicon esculentum Mill. Z Vererbungslehre 89:587-613. PMID: 1360483

Hamilton AJ, Baulcombe DC (1999) A species of small antisense RNA in posttranscriptional gene silencing in plants. Science 286:950-952. https://doi.org/10.1126/science.286.5441.950.

Hokaiwado N, Takeshita F, Banas A, Ochiya T (2008) RNAi-based drug discovery and its application to therapeutics. IDrugs 11:274-278. PMID: 18379962.

Hollick JB (2010) Paramutation and development. Annu Rev Cell Dev Biol 26:557-579. https://doi. org/10.1146/annurev.cellbio.042308.113400

Hollick J (2017) Paramutation and related phenomena in diverse species. Nat Rev Genet 18:5-23. https://doi.org/10.1038/nrg.2016.115

Hövel I, Pearson NA, Stam M (2015) Cis-acting determinants of paramutation. Sem Cell Dev Biol 44:22-32. https://doi.org/10.1016/j.semcdb.2015.08.012

Hutvagner G, Simard MJ (2008) Argonaute proteins: key players in RNA silencing. Nat Rev Mol Cell Biol 9:22-32. https://doi.org/10.1038/nrm2321.

Jiang L, Wei C, Li Y (2012) Viral suppression of RNA silencing. Sci China Life Sci 55:109-118. https:// doi.org/10.1007/s11427-012-4279-x

Johanson TM, Lew AM, Chong MMW (2013) MicroRNA-independent roles of the RNase III enzymes Drosha and Dicer. Open Biol 3:130144. https://doi.org/10.1098/rsob.130144

Martienssen R, Moazed D (2015) RNAi and heterochromatin assembly. Cold Spring Harb Perspect Biol 7:a019323. https://doi.org/10.1101/cshperspect.a019323

Matzke MA, Mosher RA (2014) RNA-directed DNA methylation: an epigenetic pathway of increasing complexity. Nat Rev Genet 15:394-408. https://doi.org/10.1038/nrg3683

Matzke MA, Primig M, Trnovsky J, Matzke AJM (1989) Reversible methylation and inactivation of marker genes in sequentially transformed tobacco plants. EMBO J 8:643-649. PMID: 16453872

Mette MF, Aufsatz W, van der Winden J, Matzke MA, Matzke AJ (2000) Transcriptional silencing and promoter methylation triggered by double-stranded RNA. EMBO J 19:5194-5201. https://doi. org/10.1093/emboj/19.19.5194

Napoli C, Lemieux C, Jorgensen R (1990) Introduction of a chimeric chalcone synthase gene into petunia results in reversible co-suppression of homologous genes in trans. Plant Cell 2:279-289. https:// doi.org/10.2307/3869076

Pikaard CS, Mittelsten Scheid O (2014) Epigenetic regulation in plants. Cold Spring Harb Perspect Biol 6:a019315. https://doi.org/10.1101/cshperspect.a019315

Romano N, Macino G (1992) Quelling: transient inactivation of gene expression in Neurospora crassa by transformation with homologous sequences. Mol Microbiol 6:3343-3353. https://doi. org/10.1111/j.1365-2958.1992.tb02202.x

Sen GL, Blau HM (2006) A brief history of RNAi: the silence of the genes. FASEB J 20:1293-1299. https://doi.org/10.1096/fj.06-6014rev

Sidorenko L, Dorweiler JE, Cigan AM, Arteaga-Vazquez M, Vyas M et al (2009) A dominant mutation in mediator of paramutation2, one of three second-largest subunits of a plant-specific RNA poly- 
merase, disrupts multiple siRNA silencing processes. PLoS Genet 5(11):e1000725. https://doi. org/10.1371/journal.pgen.1000725

Song JJ, Smith SK, Hannon GJ, Joshua-Tor L (2004) Crystal structure of Argonaute and its implications for RISC slicer activity. Science 305:1434-1437. https://doi.org/10.1126/science.1102514.

Stam M, Mol JNM, Kooter JM (1997) The silence of genes in transgenic plants. Ann Bot 79:3-12. https://doi.org/10.1006/anbo.1996.0295

Stam M, Belele C, Dorweiler JE, Chandler VL (2002) Differential chromatin structure within a tandem array $100 \mathrm{~kb}$ upstream of the maize $b 1$ locus is associated with paramutation. Genes 16:1906-1918. https://doi.org/10.1101/gad.1006702

Wassenegger M, Heimes S, Riedel L, Sänger HL (1994) RNA-directed de novo methylation of genomic sequences in plants. Cell 76:567-576. https://doi.org/10.1016/0092-8674(94)90119-8

Wei K, Wu L, Chen Y, Wang Y, Liu Y, Xie D (2013) Argonatue protein as a linker to command center of physiological processes. Chin J Cancer Res 25:430-441. https://doi.org/10.3978/j.issn.10009604.2013.08.13

Open Access This chapter is licensed under the terms of the Creative Commons Attribution 4.0 International License (http://creativecommons.org/licenses/by/4.0/), which permits use, sharing, adaptation, distribution and reproduction in any medium or format, as long as you give appropriate credit to the original author(s) and the source, provide a link to the Creative Commons license and indicate if changes were made.

The images or other third party material in this chapter are included in the chapter's Creative Commons license, unless indicated otherwise in a credit line to the material. If material is not included in the chapter's Creative Commons license and your intended use is not permitted by statutory regulation or exceeds the permitted use, you will need to obtain permission directly from the copyright holder. 